\title{
Lidil
}

Revue de linguistique et de didactique des langues

$31 \mid 2005$

Corpus oraux et diversité des approches

\section{Impact du discours adressé à l'enfant sur l'acquisition des verbes en français}

Florence Chenu et Harriet Jisa

\section{(2) OpenEdition}

\section{Journals}

Édition électronique

URL : http://journals.openedition.org/lidil/133

DOI : $10.4000 /$ lidil. 133

ISSN : $1960-6052$

\section{Éditeur}

UGA Éditions/Université Grenoble Alpes

\section{Édition imprimée}

Date de publication : 1 juin 2005

Pagination : 85-100

ISBN : 2-914176-12-0

ISSN : 1146-6480

\section{Référence électronique}

Florence Chenu et Harriet Jisa, «Impact du discours adressé à l'enfant sur l'acquisition des verbes en français », Lidil [En ligne], 31 | 2005, mis en ligne le 03 octobre 2007, consulté le 01 mai 2019. URL :

http://journals.openedition.org/lidil/133; DOI : 10.4000/lidil.133

Ce document a été généré automatiquement le 1 mai 2019.

(C) Lidil 


\title{
Impact du discours adressé à l'enfant sur l'acquisition des verbes en français
}

\author{
Florence Chenu et Harriet Jisa
}

1 Bien que l'argument de la pauvreté du stimulus (Chomsky, 1965) ait clairement mis en question le discours adressé à l'enfant comme source d'acquisition du langage, des études ont mis en évidence ses spécificités et relancent la question des caractéristiques du discours adressé à l'enfant comme facteurs d'acquisition du langage. Ces dernières années, l'impact des caractéristiques spécifiques aux langues dans l'input maternel a été l'objet de nombreuses recherches (Sampson, 1989; Goldberg, 1995; Lieven, Pine et Baldwin, 1997; O'Grady, 1997 ; Tomasello et Brooks, 1999; Cartwright et Brent, 1997). Toutefois relativement peu d'études concernent le français. Cet article se propose de contribuer à ce champ d'études en examinant 3 hypothèses (fréquence, position, diversité) concernant l'acquisition des verbes chez 2 enfants monolingues français (18-29 mois) enregistrés tous les 15 jours en interaction avec leur mère (audio/vidéo).

2 La première hypothèse est que la fréquence des verbes (types et tokens) dans l'input maternel influencerait les verbes observés dans la production de l'enfant. La deuxième hypothèse postule que la saillance perceptive du verbe dans la construction (position initiale ou finale) facilite pour l'enfant l'extraction de la forme (Naigles et Hoff-Ginsberg, 1998 ; Goldin-Meadow, Selignman et Gelman, 1976; Caselli, Bates, Casadio, Fenson, Fenson, Sardarl et Weir, 1995 ; Smiley et Huttenlocher, 1995). Enfin, la diversité des usages syntaxiques dans l'input faciliterait l'élaboration des caractéristiques syntaxiques et sémantiques des verbes (Lederer, Gleitman et Gleitman, 1995 ; Naigles, Fowler et Helm, 1995 ; Braine et Brooks, 1995 ; Maratsos et Deák, 1995).

Dans un premier temps, nous présentons la méthodologie associée au recueil et aux traitements préliminaires des données. Nous développons ensuite chacune des trois hypothèses.

Recueil et traitements préliminaires des données 
Pour cette étude, nous utilisons des données naturelles issues d'un large corpus initialement entrepris pour l'étude du développement phonologique et morphologique chez les enfants francophones' (Hilaire-Debove et Kehoe, sous presse ; Kehoe et HilaireDebove, sous presse; Rose et Dos Santos, sous presse). Ce corpus est actuellement en cours de constitution. 8 dyades mère-enfant ${ }^{2}$ ont été recrutées pour des séances d'enregistrement vidéo bi-mensuelles à leur domicile deux années durant (enfant âgé de 12 à 36 mois). Les premiers enregistrements ont eu lieu en mars 2002; les derniers enregistrements ont eu lieu en octobre $2004^{3}$.

$5 \quad$ Les enregistrements se déroulent au domicile de la dyade. L'expérimentateur se présente avec une caméra numérique et deux micros HF. Le boîtier HF du micro destiné à l'enfant est dissimulé dans un petit sac à dos en forme d'ours en peluche. L'enregistrement dure une heure sauf si les conditions ne le permettent pas (enfant fatigué, malade...). L'expérimentateur a pour consigne de ne pas intervenir dans les discussions.

6 Les transcriptions sont effectuées par des étudiants rémunérés et formés à la transcription selon les conventions de base de CHILDES (MacWhinney, 1995) légèrement adaptées aux besoins des études en cours. Pour nous, le choix de cHILDEs répond à plusieurs exigences: il s'agit d'un outil adapté à l'étude de données naturelles en interaction; il comporte des modules développés pour l'analyse de la morphologie et de la syntaxe; le système est assez souple pour permettre des codages et analyses spécifiques. Le logiciel CHILDES présente des inconvénients (commandes parfois fastidieuses, ergonomie pauvre, multiplication des fichiers pour la génération des codages et des analyses, bugs, etc.). Cependant, outre le coût qu'aurait impliqué le développement d'outils spécifiques à l'analyse de nos corpus, il nous semble important d'utiliser les outils existants pour deux raisons principales: si un outil est utilisé par la communauté des chercheurs il est probable que les efforts se mutualisent pour son amélioration et son maintien (mise à jour, adaptation aux technologies nouvelles...) ; cela facilite la réutilisabilité des données et impose un format commun assurant une meilleure comparabilité des données.

7 Pour cette étude, 3 transcriptions pour chacun des deux enfants ont été sélectionnées. Le tableau 1 présente pour chaque enregistrement, l'âge de l'enfant, le nombre d'énoncés produits par la mère et par l'enfant ainsi que la longueur moyenne d'énoncé (LME) pour l'enfant.

\begin{tabular}{|l|l|l|l|l|}
\hline & MAR & MèRE DE MAR & THE & MêRE DE THE \\
\hline \hline Âge en mois & 18 & & 18 & \\
\hline Total énoncés & 401 & 746 & 384 & 718 \\
\hline \hline LME & 1.3 & 1.3 & & \\
\hline Âge en mois & 24 & & 24 & \\
\hline \hline Total énoncés & 707 & 698 & 500 & 836 \\
\hline \hline LME & 2.68 & & 2.18 & \\
\hline
\end{tabular}




\begin{tabular}{|l|l|l|l|l|}
\hline Âge en mois & 29 & & 29 & \\
\hline \hline Total énoncés & 687 & 786 & 740 & 823 \\
\hline \hline LME & 3.7 & & 2.9 & \\
\hline
\end{tabular}

Tableau 1 - Participants : MAR et THE ; 18, 24 et 29 mois ; nombre total d'énoncés et longueur moyenne des énoncés.

Fréquence des verbes dans l'input

8 La fréquence est un paramètre important pour les verbes, dans la mesure où la réalité qu'ils dénotent n'est pas appréhendable aussi immédiatement que pour les noms concrets (Gentner, 1982). Pour extraire le sens d'un verbe, un grand nombre d'occurrences est nécessaire. Nous examinons ici les relations existant entre la fréquence des verbes dans l'input maternel et les verbes observés dans les productions de l'enfant. On s'attend à ce que les verbes les plus fréquents dans l'input connaissent un avantage d'acquisition.

Une étape de lemmatisation partiellement automatisée (désambiguïsation manuelle) à l'aide de la version française de la commande «mor» de CLAN (Parisse et Le Normand, $2000)^{4}$ a été réalisée. Elle permet d'obtenir pour chaque mot sa classe morphologique ainsi que des informations sur les morphèmes flexionnels qui en font partie ainsi que l'illustre l'exemple (1). Doudou est associé à la classe des «noms propres » (n: prop) et mange à la classe des «verbes» (v). Le lemme des items lexicaux variables est, pour les verbes la forme infinitive, pour les noms la forme singulier, pour les adjectifs la forme masculin singulier. Les informations grammaticales portées par ces variations sont indiquées à la suite du lemme. Ainsi, dans l'exemple (1) «\& PRES \& 3SV » indique que l'occurrence est la forme d'indicatif présent à la troisième personne du singulier.

\begin{tabular}{|l|l|l|l|}
\hline$(1)$ & *SBJ: & Doudou & mange \\
\hline \hline & \%mor: & n: prop|Doudou & v/manger\&PRES\&3SV \\
\hline
\end{tabular}

Les commandes de CHILDES permettent ensuite d'établir des listes de fréquences en fonction de critères précis (mots, catégories, lemmes...).

Les listes de fréquence de types de verbes chez la mère et l'enfant ainsi obtenues permettent de calculer les cœefficients de corrélation de Spearman ${ }^{5}$ présentés dans les tableaux 2 et 3.

\begin{tabular}{|l|l|l|l|}
\hline & MAR 18 mois & MAR 24 mois & MAR 29 mois \\
\hline \hline Mère & $r=0.309167^{6}$ & $r=0.34944^{7}$ & $r=0.593190^{7}$ \\
\hline
\end{tabular}

Tableau 2 - Corrélations de rangs sur tous les verbes pour le couple MAR/Mère.

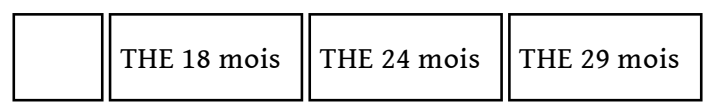


\begin{tabular}{|l|l|l|l|}
\hline Mère $r=0.179537^{6}$ & $r=0.472889^{7}$ & $r=0.539250^{7}$ \\
\hline
\end{tabular}

Tableau 3 - Corrélations de rangs sur tous les verbes pour le couple THE / Mère.

12 Les corrélations ne sont pas fortes, mais augmentent avec le temps. De façon générale, les verbes les plus fréquents dans l'input sont observés dans la production de l'enfant. Il s'agit, pour une large part de verbes « légers » (Brugman, 2001) tels que être, avoir, aller, mettre, regarder, vouloir, prendre, etc. c'est-à-dire de verbes dont la sémantique est peu restrictive et leur permet de convenir à la dénotation de situations diverses.

13 La préférence pour les verbes « légers » répond aux maximes de quantité de Grice (Grice, 1975) : «Rendez votre contribution aussi informative que cela est requis (pour les objectifs immédiats de l'échange)» et « Ne rendez pas votre contribution plus informative que nécessaire "». Le tableau (4) présente des exemples d'emplois du verbe mettre issus de nos corpus dans lesquels auraient pu figurer les verbes plus « lourds » de la dernière colonne.

\begin{tabular}{|l|l|l|}
\hline Exemple & Situation typique & $\begin{array}{l}\text { vb. } \\
\text { alternatif }\end{array}$ \\
\hline \hline a. i met des chaussettes & enfilant des chaussettes. & enfiler \\
\hline \hline b. faut qu(e) tu l(e) mettes là & assemblant des legos, & emboîter, \\
\hline \hline & des pièces de puzzle. & insérer \\
\hline \hline c. si tu mets que la tête dans & bourrant un gros jouet & enfoncer, \\
\hline \hline \begin{tabular}{l|l} 
la poussette ça tiendra \\
jamais
\end{tabular} & $\begin{array}{l}\text { dans une poussette, bourrant une serviette dans un } \\
\text { verre. }\end{array}$ & bourrer \\
\hline \hline d. tu l'mets dans la tasse? & versant du liquide dans une tasse. & verser \\
\hline \hline e. tu mets ta veste là ? & $\begin{array}{l}\text { accrochant un manteau à une patère, un wagon à } \\
\text { un autre. }\end{array}$ & accrocher \\
\hline
\end{tabular}

Tableau 4 - Exemples d'emplois de mettre répondant aux maximes de Grice.

14 Les mères utilisent toutefois sporadiquement des verbes «lourds » lorsqu'elles désirent attirer l'attention de l'enfant sur un aspect spécifique de la situation (Slobin, 1997). Par exemple, après de multiples répétitions de (e) et les échecs répétés de l'enfant à suspendre sa veste à une patère, la mère produit (2).

(2) accroche-la comme il faut !

15 Les principes de Grice devraient également s'appliquer au français oral entre adultes et la fréquence de ces verbes ne serait alors pas spécifique au discours adressé à l'enfant. Toutefois, la corrélation entre le corpus Gougenheim (CG) ${ }^{10}$ (Gougenheim, Michéa, Rivenc et Sauvageot, 1964) et nos données n'est pas très importante $(r=0.6292 ; p<0.00001)$ et 
indique une spécificité du discours adressé à l'enfant. Les verbes les plus fréquents de nos corpus pour lesquels des différences significatives $(\mathrm{p}<0.05)$ s'observent par rapport à CG sont répertoriés dans le tableau 5 . La partie supérieure du tableau présente les verbes significativement plus employés dans nos données, tandis que la partie inférieure présente ceux qui sont significativement plus employés dans CG.

\begin{tabular}{|c|c|c|c|}
\hline Verbe & différence & $\mathrm{z}$ & $\mathrm{p}<$ \\
\hline mettre & 0.033 & 20.08922722 & 0.0001 \\
\hline aller & 0.030 & 10.38740536 & 0.0001 \\
\hline regarder & 0.026 & 24.65607717 & 0.0001 \\
\hline vouloir & 0.023 & 11.52574241 & 0.0001 \\
\hline être & 0.017 & 2.458152148 & 0.05 \\
\hline faire & 0.017 & 4.667642688 & 0.0001 \\
\hline jouer & 0.010 & 11.46723467 & 0.0001 \\
\hline tenir & 0.008 & 7.344627646 & 0.0001 \\
\hline manger & 0.008 & 7.764239977 & 0.0001 \\
\hline attendre & 0.008 & 9.278872744 & 0.0001 \\
\hline enlever & 0.006 & 10.68389825 & 0.0001 \\
\hline appuyer & 0.005 & 14.13500542 & 0.0001 \\
\hline savoir & -0.012 & -5.148219089 & 0.0001 \\
\hline dire & -0.024 & -7.730265161 & 0.0001 \\
\hline avoir & -0.064 & -10.1285108 & 0.0001 \\
\hline
\end{tabular}

Tableau 5 - Liste des principaux verbes significativement différents entre nos données et CG.

Les verbes significativement plus employés dans nos données sont parmi les premiers produits par l'enfant. Il s'agit de verbes utilisés par les mères pour établir l'attention conjointe (regarder), négocier les intentions (vouloir), dénoter les activités (faire), les déplacements (aller), les actions de manipulation simple (mettre, tenir, enlever, appuyer...). On trouve également dans cette liste quelques verbes appartenant aux champs lexicaux associés aux activités typiques des enfants (jouer; manger, etc.). Les différences pour être et avoir doivent recevoir d'autres interprétations". Être dans nos corpus est fréquemment et majoritairement employé par les mères lorsqu'elles identifient ou localisent des entités 
appartenant à la situation de communication. Avoir, en revanche, est significativement plus présent dans CG et cela est probablement le fait d'une utilisation du passé composé relativement plus faible chez les mères dans nos corpus.

Il y a évidemment beaucoup de verbes hapax dans le discours des mères. Nos mesures prennent en compte des fréquences relatives et permettent d'examiner les verbes proportionnellement plus fréquents chez les mères et chez les enfants et de faire ressortir les spécificités du discours de chaque participant

Parmi les verbes proportionnellement plus fréquents de façon significative dans le discours maternel que dans le discours enfantin, se trouvent des auxiliaires (ÊTRE, AVOIR), des verbes utilisés pour attirer l'attention de l'enfant sur des objets et/ou des caractéristiques des objets (VOIR, POUVOIR, APPELER, ex. Tu vois le cerceau ? Enfin bon, on peut ouvrir l(e) couvercle., Comment $t(u)$ appelles ça toi ?), des verbes pour diriger l'action de l'enfant (ATTENDRE, FALLOIR, ex. Attends!, Faut croquer fort.), solliciter des reformulations ou s'assurer de la compréhension (DIRE, ex. Tu dis quoi ? C'est ça que tu m(e) dis ?). Parmi les verbes proportionnellement plus fréquents de façon significative dans le discours enfantin, se trouvent des verbes pour exprimer le résultat d'actions (CASSER, CACHER, FINIR, ex. Cassé, Caché, On a fini.), des actions à venir (DESSINER, EFFACER, ex. Dessiner encore., Veux effacer.), des verbes accompagnant des actions (ALLER, ARRIVER, ex. Ça ça va là., Arrive pas moi.).

Position des verbes, diversité des constructions dans l'inputPosition des verbes

Pour ce qui concerne la position l'hypothèse est que la position (initiale ou finale) du verbe dans la construction facilite pour l'enfant l'extraction de la forme (Naigles et HoffGinsberg, 1998; Goldin-Meadow, Selignman et Gelman, 1976; Caselli, Bates, Casadio, Fenson, Fenson, Sardarl et Weir, 1995; Smiley et Huttenlocher, 1995). Les référents nouveaux en français parlé sont typiquement introduits par un item lexical en position post-verbale puis repris par des clitiques avant le verbe dans les propositions suivantes, ce qui n'est pas le cas pour l'anglais où l'argument pronominalisé reste dans sa position post-verbale. Ainsi, il est possible, en français parlé, que le verbe se trouve souvent dans une position finale saillante. Il convient donc dans un premier temps d'examiner si tel est le cas dans nos corpus.

Un deuxième niveau de codage a donc été établi pour la position du verbe, celle de ses arguments et des autres constituants de la phrase. Les diagrammes (1-4) présentent les proportions de verbes selon la position dans les constructions maternelles contenant des verbes. La position finale ne semble pas une position privilégiée par les mères. 

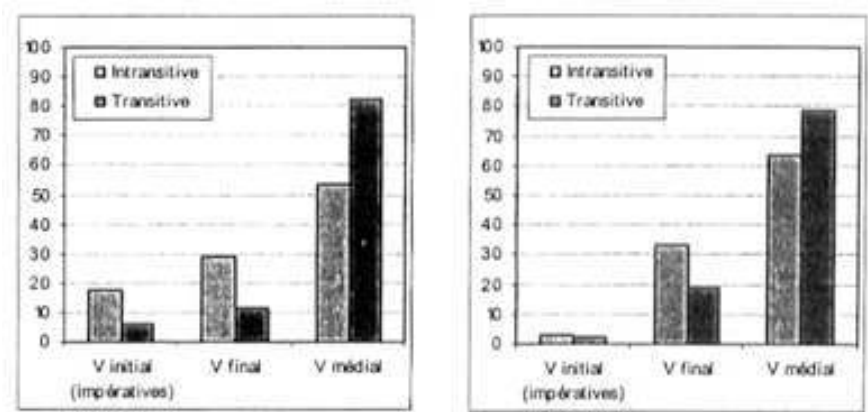

Diagramme 1 - MAR 24 mois: position des verbes dans I'input ( $\%$ ). Diagramme 2 - MAR 29 mois: position des verbes dans l'input (\%)
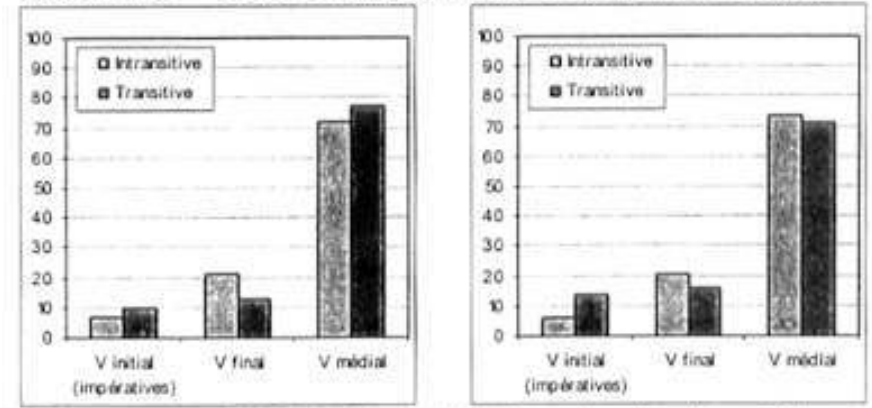

Diagramme 3 - THE 24 mois: position des verbes dans l'input (\%). Diagramme 4 - THE 29 mois: position des verbes dans l'input (\%).

En effet, contrairement à ce qui était attendu, la position préférée pour le verbe dans le discours maternel est au milieu de la construction. Ainsi les mères semblent-elles éviter la pronominalisation de l'objet dans les constructions transitives. Si elles pronominalisent l'objet, c'est préférentiellement à l'aide de déictiques (ça, celui-là etc.) qui ne subissent pas le déplacement en position préverbale. Le discours des mères est également chargé de constructions copules (SN|pro est SN|adj|SP) ${ }^{12}$.

Il reste à savoir si, malgré la faible fréquence des positions initiale et finale, les verbes qui y entrent connaissent un avantage d'acquisition et si cet avantage évolue développementalement. Les diagrammes (5-6) présentent le pourcentage de verbes (types) produits par l'enfant en fonction de la position utilisée par la mère, ie. seulement en position initiale ${ }^{13}$, seulement en position finale, seulement en position médiale, ou dans des positions diverses.
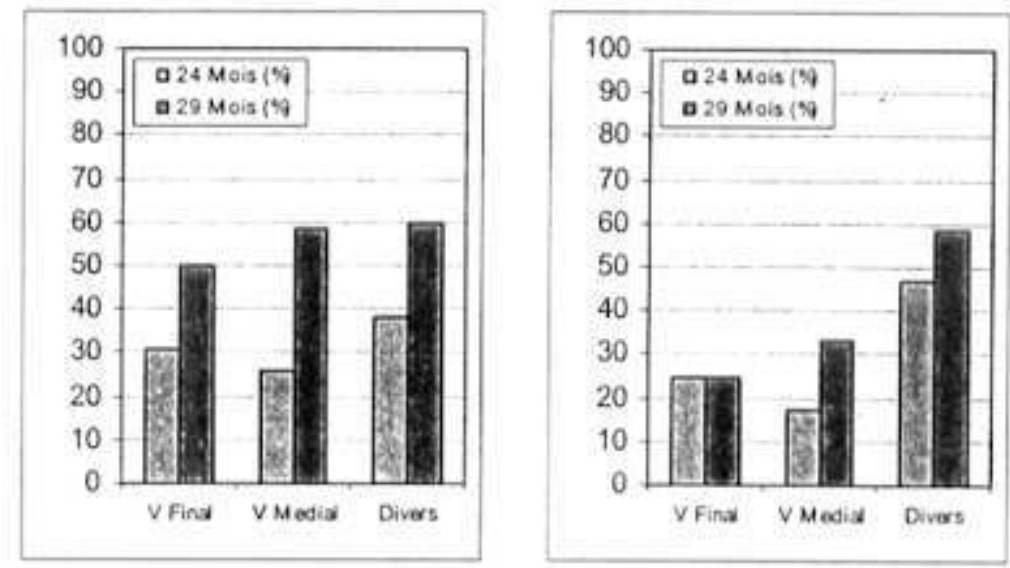

Diagramme 5 - Verbes produits par MAR en fonction de la position dans l'input (\%) Diagramme 6 - Verbes produits par THE en fonction de la position dans l'input (\%) 
Pour les deux enfants, à 24 et à 29 mois, les verbes qui apparaissent dans des contextes diversifiés présentent un léger avantage d'acquisition, statistiquement significatif $(\mathrm{p}<$ 0.0005). Les différences de proportions qui apparaissent sur les graphiques entre 24 et 29 mois ne sont pas significatives, excepté pour les verbes en position finale chez MAR $(\mathrm{z} \approx$ $2.35 ; \mathrm{p}<0.05)$. Ces résultats sont conformes à ceux obtenus par Naigles et Hoff-Ginsberg (1998) et permettent d'argumenter en faveur de l'hypothèse selon laquelle la diversité des constructions d'un verbe permet à l'enfant d'augmenter ses connaissances sur ce dernier. Diversité des constructions

24 Nous prédisons ici que la diversité des usages syntaxiques dans l'input facilite l'élaboration des caractéristiques syntaxiques et sémantiques des verbes (Lederer, Gleitman et Gleitman, 1995 ; Naigles, Fowler et Helm, 1995; Braine et Brooks, 1995 ; Maratsos et Deák, 1995). Bien que l'ordre des mots pour le français soit caractérisé comme SVO, le français parlé présente une variation considérable. Une source de diversité possible est la dislocation puisque cette dernière peut, sans modifier le schème argumental, réarranger l'ordre des mots ainsi que l'illustrent les exemples suivants (3-10).

\begin{tabular}{|l|l|}
\hline Dislocation sujet à gauche & Dislocation objet à gauche \\
\hline \hline 3. Pierre $_{i} \boldsymbol{i l}_{i}$ voit le chat. & 5. Le chat $_{i}$ Pierre $\boldsymbol{l}_{i}$ voit. \\
\hline \hline 4. Lui $_{i} \boldsymbol{i l}_{i}$ voit le chat. & 6. Lui $_{i}$ Pierre $\boldsymbol{l}_{i}$ voit. \\
\hline
\end{tabular}

\begin{tabular}{|c|c|}
\hline Dislocation sujet à droite & Dislocation objet à droite \\
\hline 7. $I_{i}$ voit le chat Pierre $_{i}$. & 9. Pierre le $e_{i}$ voit le chat ${ }_{i}$. \\
\hline 8. $I_{i}$ voit le chat lui $i_{i}$. & 10. Pierre le $\boldsymbol{e}_{i}$ voit lui ${ }_{i}$. \\
\hline
\end{tabular}

La dislocation est réputée fréquente en français parlé (Blanche-Benveniste, 1997; Lambrecht, 1994). On s'attend donc à ce qu'elle joue également un rôle dans le discours adressé à l'enfant. Les types de dislocations susceptibles de réarranger l'ordre des mots en français sont la dislocation objet gauche (O S cl-V) et la dislocation sujet droite (cl-V o S). Le diagramme 7 présente la proportion, dans l'input maternel, de dislocations et d'ordre canonique simple des arguments sujet et objet lorsque le verbe est en position médiale. 


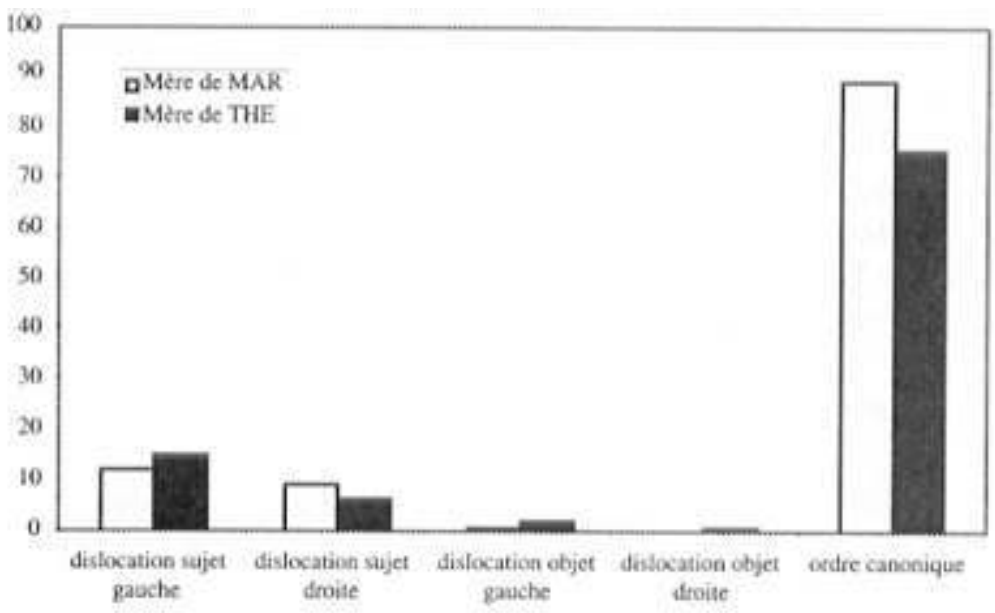

Diagramme 7 - Dislocations dans les constructions à verbe médial dans l'input (\%).

L'ordre canonique est très largement préféré par les mères lorsque le verbe est en position médiale dans nos corpus

La première hypothèse nous a conduit à examiner la relation entre les verbes fréquents dans le discours maternel et l'acquisition de ces verbes par l'enfant. De façon générale, les verbes les plus fréquents chez la mère sont acquis par l'enfant et cette tendance s'accentue avec le temps. Toutefois, l'étude de la fréquence relative a mis en évidence des différences de fréquences relatives indiquant des spécificités des discours maternel et enfantin. Ainsi les verbes pour attirer l'attention de l'enfant, lui présenter des caractéristiques des objets et solliciter son interaction sont proportionnellement plus représentés dans le discours maternel. Dans le discours de l'enfant, les verbes significativement plus importants sont des verbes cognitivement saillants pour l'enfant c'est-à-dire utilisés pour parler de ses actions accomplies ou à venir (Bassano, 2000).

Notre hypothèse qui prédisait que les verbes en position finale seraient acquis précocement à cause de la saillance de cette position n'est pas confirmée. Il s'avère que les verbes utilisés dans des positions diverses (y compris la position finale) sont acquis précocement par l'enfant. Il est évident que plus un verbe est fréquent, plus il porte un potentiel d'être utilisé dans des positions diverses, ce qui complémente notre première hypothèse.

30 Finalement, nous avons examiné l'utilisation des constructions disloquées comme étant une source possible de variabilité de la position du verbe. Contrairement à ce qui est observé dans l'interaction adulte-adulte, nos deux mères semblent éviter les dislocations, préférant l'ordre canonique SVO.

31 Nous avons entrepris ce travail dans le but d'examiner comment le discours adressé à l'enfant influence l'acquisition des verbes. Les résultats présentés ici suggèrent fortement que la diversité d'utilisation des verbes facilite leur acquisition (Naigles et Hoff-Ginsberg, 1998). Ils suggèrent également que les constructions disloquées ne contribuent pas beaucoup à cette diversité. Nous voulons poursuivre notre investigation en regardant en détail la variété des constructions utilisées par les mères avec l'hypothèse que la variété donne à l'enfant des indices syntaxiques et sémantiques pour l'acquisition des verbes. 


\section{BIBLIOGRAPHIE}

BASSANO, D., 2000, « Early development of nouns and verbs in French: exploring the interface between lexicon and grammar ", in Journal of Child Language, Vol. 27:3, 521-559.

BLANCHE-BENVENISTE, C., 1997, Approches de la langue parlée en français, Ophrys, Gap, Paris.

BRAINE, M. D. S. ET BROOKS, P. J., 1995, « Verb argument structures and the problem of avoiding an overgeneral grammar ", in Beyond Names for Things: Young Children's acquisition of Verbs, M. Tomasello et W. E. Merriman (eds), Erlbaum, Hillsdale, N. J., 353-376.

BRUGMAN, C., 2001, « Lights verbs and polysemy », in Language Sciences, Vol. 23 : 4-5, 551-578.

CARTWRIGHT, T.A. et BRENT, M.R., 1997, « Syntactic categorization in early language acquisition: formalizing the role of distributional analysis », in Cognition, Vol. 63, 121-170.

CASELLI, M. C., BATES, E., CASADIO, P., FENSON, J., FENSON, L., SANDERL, L. et WEIR, J., 1995, « A crosslinguistic study of early lexical development », in Cognitive Development, Vol. 10:2, 159-199. CHомSкY, N., 1965, Aspects of the theory of syntax, MIT press, Cambridge Mass.

COLÉ, P.et MORGAN, J. L. (eds), 1975, Syntax and Semantics Vol. 3 Speech acts, Academic Press, New York.

GENTNER, D., 1982, « Why nouns are learned before verbs: Linguistic relativity versus natural partitioning ", in Language development, S. A. Kuczaj (ed), Lawrence Erlbaum Associates, Hillsdale, N.J., 301-334.

GOLDBERG, A.E., 1995, Constructions: a construction grammar approach to argument structure, University of Chicago Press, Chicago.

GOLDIN-MEADOW, S., SELIGMAN, M. E. P. et GELMAN, R., 1976, « Language in the two-year old: Receptive and productive stages $»$, in Cognition, Vol. 4:2, 189-202.

GOUGENHEIM, G., MICHÉA, R., RIVENC, P. et SAUVAGEOT, A., 1964, L'élaboration du français fondamental (1 ${ }^{\text {er }}$ degré) : étude sur l'établissement d'un vocabulaire et d'une grammaire de base, Didier, Paris.

GRICE, H.P., 1975, « Logic and conversation », in Syntax and Semantics Vol. 3 Speech acts, P. colE et J. L. MORGAN (eds), Academic Press, New York, 41-58.

HILAIRE-DEBOVE, G. et KeHOE, M., (sous presse), « Acquisition des consonnes finales (codas) chez les enfants francophones : des universaux aux spécificités de la langue maternelle », $X X V^{e}$ Journées d'étude sur la parole, Fez (Maroc).

HOWELL, D.C., 1998, Méthodes statistiques en sciences humaines, DeBoeck Université.

KEHOE, M. et HILAIRE-DEBOVE, G., (sous presse), « The structure of branching onsets and rising diphtongs: Evidence from acquisition ", Proceedings of 28th annual Boston University Conference on Language Development, October 2003, Boston, USA.

KUCZAJ, S.A. (ed) 1982, Language development: language, thought, and culture, Lawrence Erlbaum Associates, Hillsdale, N.J.

LAMBRECHT, K., 1994, Information structure and sentence form: topic, focus, and the mental representations of discourse referents, Cambridge University Press, Cambridge, New York. 
LEDERER, A., GLEITMAN, H. et GLEITMAN, L. R., 1995, « Verbs of a feather flock together: Semantic information in the structure of maternal speech », in Beyond Names for Things: Young Children's acquisition of Verbs, M. Tomasello et W. E. Merriman (eds), Erlbaum, Hillsdale, N.J., 277-297.

LIEVEN, E., PINE, J. et BALDWIN, G., 1997, « Lexically-based learning and early grammatical development », in Journal of Child Language, Vol. 24:1, 187-219.

MACWHINNEY, B., 1995, The CHILDES project: tools for analyzing talk, Erlbaum, Hillsdale, N.J.

MARATSOS, M. et DEÁK, G., 1995, « Hedgehogs, foxes, and the acquisition of verb meaning », in Beyond Names for Things: Young Children's acquisition of Verbs, M. Tomasello et W. E. Merriman (eds), Erlbaum, Hillsdale, N.J., 377-404.

NAIGLES, L.R., FOWLER, A. et HELM, A., 1995, « Syntactic bootstrapping from start to finish with special reference to Down syndrome ", in Beyond Names for Things: Young Children's acquisition of Verbs, M. Tomasello et W. E. Merriman (eds), Erlbaum, Hillsdale, N.J., 299-330.

NAIGLES, L.R. et HOFF-GINSBERG, E., 1998, « Why are some verbs learned before other verbs. Effects of input frequency and structure on children's early verb use », in Journal of Child Language, Vol. 25:1, 95-120.

O'GRADY, W., 1997, Syntactic development, University of Chicago Press, Chicago.

PARISSE, C. et LE NORMAND, M.-T., 2000, « Automatic disambiguation of morphosyntax in spoken language corpora ", in Behavior Research Methods, Instruments and Computers, Vol. 32 : 3, 468-481. ROSE, Y. et DOS SANTOS, C., sous presse, «Effets positionnels dans l'acquisition du français », $X X V^{e}$ Journées d'étude sur la parole, Fez (Maroc).

SAMPSON, G., 1989, « Language acquisition: growth or learning? » in Philosophical Papers, Vol. 18, 203-240.

SLOBIN, D.I. (ed) 1997, The crosslinguistic study of language acquisition: Vol. 5. Expanding the contexts, Erlbaum, Hillsdale, N.J.

SLOBIN, D.I., 1997, «The origins of grammaticizable notions: beyond the individual mind ", in The crosslinguistic study of language acquisition : Vol. 5. Expanding the contexts, D.I. Slobin (ed), Erlbaum, Hillsdale, N.J., 265-323.

SMILEY, P. A. et HUTTENLOCHER, J., 1995, « Conceptual development and the child's early words for events, objects and persons ", in Beyond names for things: Young children's acquisition of verbs, M. Tomasello et W. E. Merriman (eds), Lawrence Erlbaum Associates, Hillsdale, NJ, 21-61.

TOMASELLO, M. et MERRIMAN, W.E. (eds), 1995, Beyond names for things: young children's acquisition of verbs, Erlbaum, Hillsdale, N.J.

TOMASELLO, M. et BROOKS, P. J., 1999, « Early syntactic development: A Construction Grammar approach ", in The Development of Language, M. D. Barrett (ed), Psychology Press, Hove, East Sussex, UK, 161-190.

TOMASELLO, M. et BATES, E. (eds), 2001, Language development. The essential readings, Essential readings in developmental psychology, Blackwell, oxford. 


\section{NOTES}

1. National Institute of Mental Health Grant \#1R0 IMH60922-01A2, projet déposé par K. Demuth (Université de Brown, Providence) 2001-2005 et ACI Terrain Techniques Théories (2003-2005 - Projet déposé par Harriet Jisa). Nous remercions vivement les parents et enfants qui ont accepté de participer à cette étude.

2. Les mères ont un niveau d'étude supérieur ou égal à Bac +3.

3. Certaines familles ont toutefois donné leur accord pour des enregistrements mensuels pour une année supplémentaire.

4. Quelques aménagements ont été apportés à la version disponible sur le site de CHILDES (homogénéisation de marqueurs, corrections orthographiques, ajout d'items).

5. Pour les notions statistiques, voir Howell (1998).

6. N.s.

7. $\mathrm{P}<0.001$

8. Les différences de corrélation ne sont pas significatives sauf pour MAR entre 24 mois et 29 mois $(p<0.05)$.

9. «Make your contribution as informative as is required (for the current purposes of the exchange). Do not make your contribution more informative than is required.»

10. Ce corpus, malgré son ancienneté, a été choisi pour sa qualité technique (corpus d'entretiens, mots étiquetés pour la catégorie grammaticale).

11. Le corpus Gougenheim ne présente pas de distinction entre les emplois pleins et les emplois auxiliaires de être et avoir. Pour la comparaison avec ce corpus, nous les avons donc également rassemblés dans nos données.

12. $\mathrm{SN}=$ Syntagme Nominal $;$ Pro = Pronom $; \operatorname{adj}=$ Adjectif $; \mathrm{SP}=$ Syntagme Prépositionnel ; | marque la disjonction.

13. Les verbes employés par la mère seulement en position initiale sont très peu nombreux, et aucun n'est produit par l'enfant.

14. $\mathrm{S}=$ Sujet $; \mathrm{O}=$ Objet $; \mathrm{V}=$ Verbe $; \mathrm{cl}=$ Clitique .

\section{AUTEURS}

\section{FLORENCE CHENU}

Institut des Sciences cognitives (UMR 5015).

HARRIET JISA

Dynamique du langage (UMR 5596) \& Université Lyon 2 